\title{
Use of limbal ring-rod for radiological localisation of ocular foreign body
}

\author{
POON KWONG-YAM \\ From Hong Kong Government Ophthalmic Service
}

SUMmARY The limbal ring-rod and the proportional geometric drawing method provide a simple, inexpensive, and fairly accurate measure for the radiological localisation of ocular foreign bodies, and the results simulate those of orbital tomography.

$X$-raying the orbit, giving frontal and lateral views with the limbal ring,,$^{1-6}$ is a simple procedure for the diagnosis and localisation of ocular foreign bodies. However, misinterpretation is not uncommon because of the $x$-ray magnification, the measuring method, and the direction of gaze ${ }^{127}$ of the examined eye. To obtain a more accurate localisation a limbal ring-rod and a measuring method comprising proportional geometric drawing have been used in the Government Ophthalmic Service since June 1987.

\section{Material and methods}

The limbal ring-rod consists of a limbal ring with a perpendicular rod whose length equals the diameter of the ring. The diameter of the ring is half the diameter of the schematic eye $\mathrm{e}^{1248}$ and is $12 \mathrm{~mm}$ in length (Fig. 1).

Correspondence to Dr Poon Kwong-yam, Yaumatei Ophthalmic Centre, 145 Battery Street, Yaumatei, Kowloon, Hong Kong.

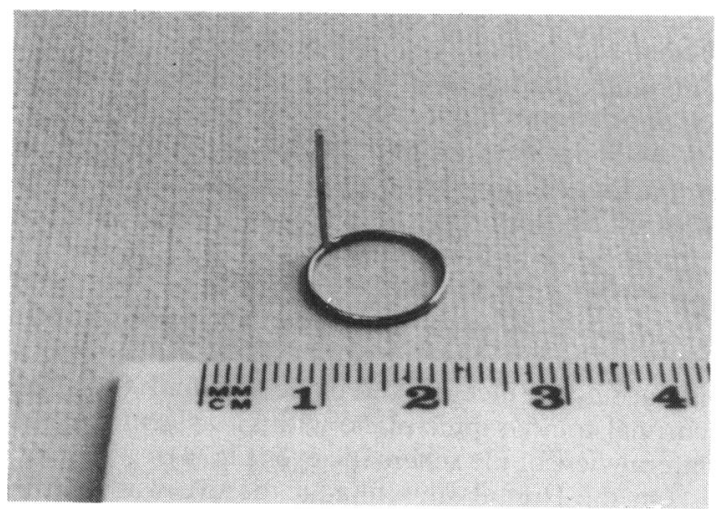

Fig. 1 Limbal ring-rod.

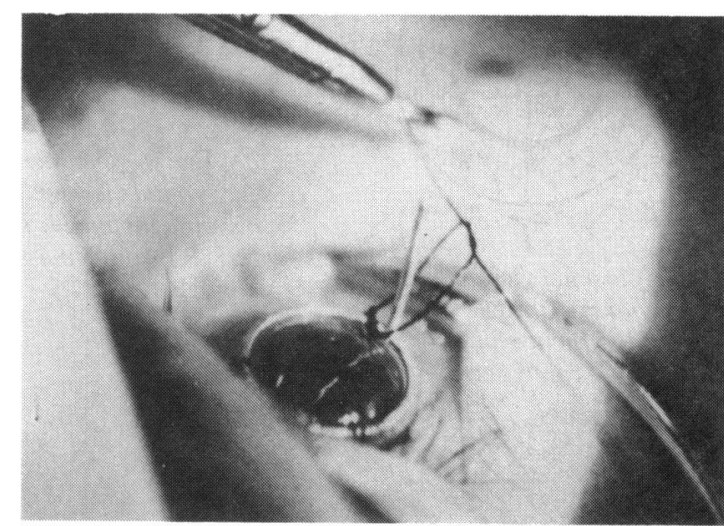

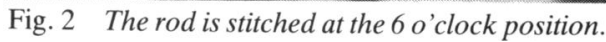

INSERTION OF LIMBAL RING-ROD

The limbal ring-rod is sutured onto the limbus with the rod fixed at the 6 o'clock position (Fig. 2). The eye can close and move freely while an $x$-ray is being taken (Fig. 3).

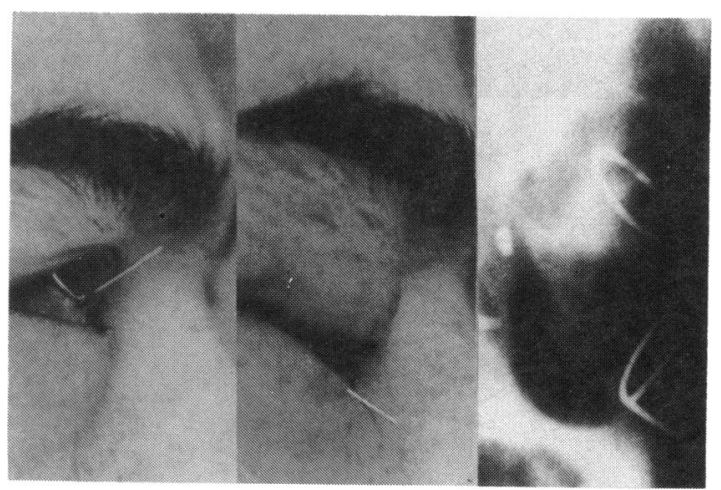

Fig. 3 The eye can move freely. 


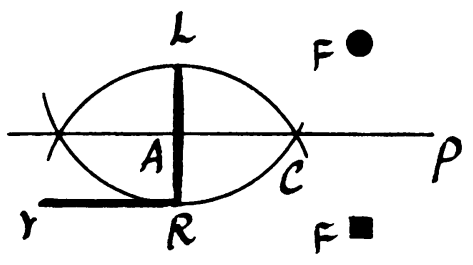

Fig. 4 Geometric construction on lateral view. AP is a bisector of the image of the limbal ring LR. C is the centre of the schematic eye in proportion.

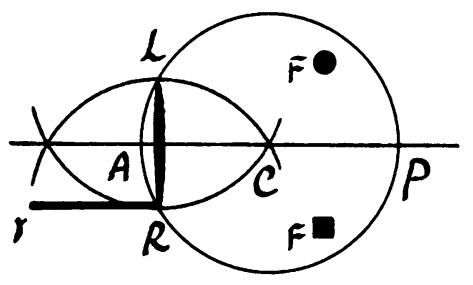

Fig. 5 The greatest circumference of the schematic eye in lateral view is produced with centre at $\mathrm{C}$ and radius $\mathrm{CL}(\mathrm{CL}=\mathrm{CR}=\mathrm{LR})$.

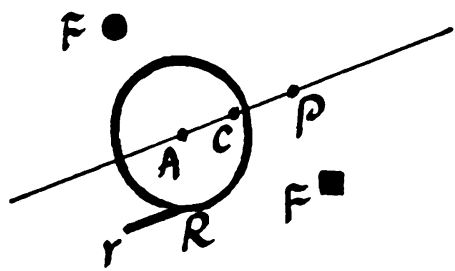

Fig. 8 Geometric construction of frontal view with the eye is not looking straight ahead. AP is parallel to the rod $\mathrm{rR}$ through the centre of the ring. $\mathrm{AP}=$ $2 \mathrm{rR}$.

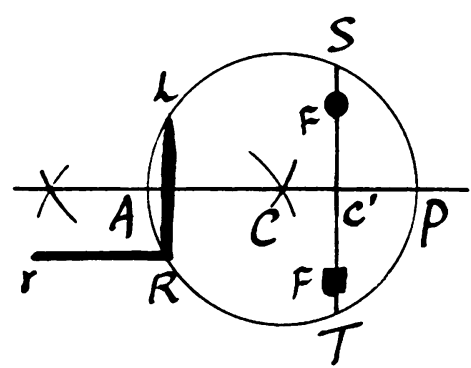

Fig. 6 A line ST is drawn through the image of the foreign body $\mathrm{F}(\mathrm{s})$ perpendicular to AP. ST is a tomographic plane in lateral view. $\mathrm{C}^{\prime}$ is the centre of this tomographic plane.

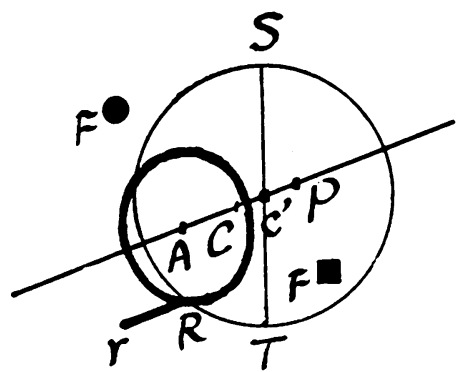

Fig. $9 \mathrm{AC}^{\prime}$ : $\mathrm{AP}$ is identical to that in the lateral view as in Fig. 6. ST is a coronal tomographic plane with $\mathrm{C}^{\prime}$ as centre and radius $\mathrm{SC}^{\prime}$ measured from the lateral view as in Fig. 6.

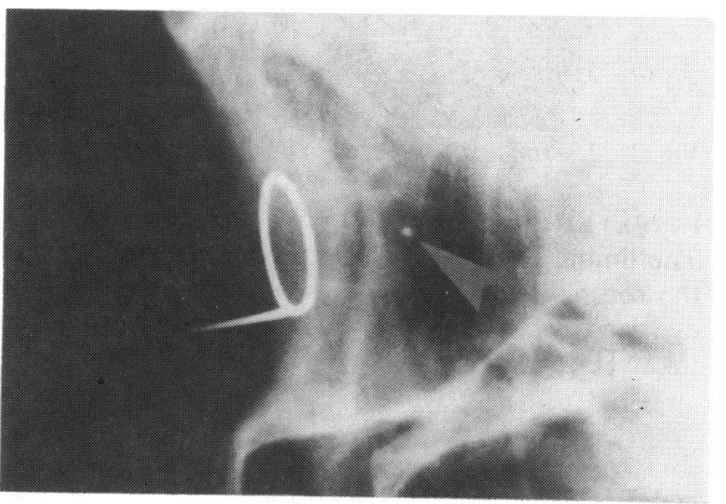

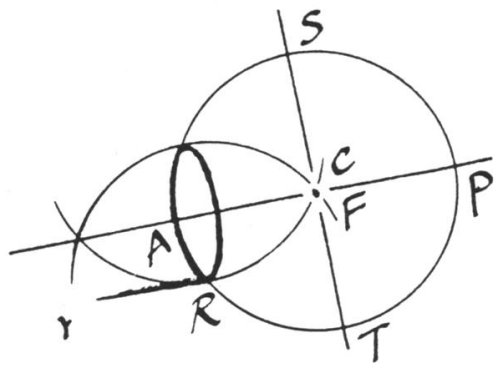

Fig. 11 The foreign body $\mathrm{F}$ is situated near the equator.
Fig. 10 In Fig. 9, if the limbal ring was used without the rod, the intraocular foreign body would be misinterpreted as an extraocular foreign body, and vice versa.

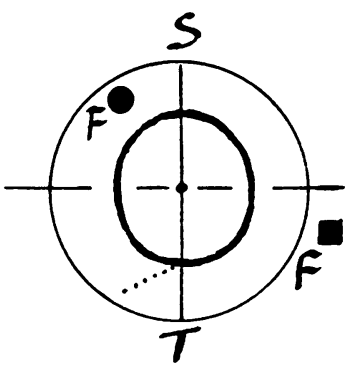

PROPORTIONAL GEOMETRIC DRAWING

A geometric drawing is made on the films directly or on paper copies of the films.

On the lateral view film the perpendicular bisector of the ring LR gives the corneal axis AP (Fig. 4). The greatest circumference of the schematic eye in lateral view is produced with centre at $C$ and radius $C L$ $(\mathrm{CL}=\mathrm{CR}=\mathrm{LR})$ (Fig. 5).

A line is drawn through the image of the foreign body (or bodies) $F(s)$ perpendicular to AP, intersecting at $C^{\prime}$ and meeting the circle at $S$ and T. ST is a coronal tomographic plane with $\mathrm{C}^{\prime}$ as centre in the lateral view of the schematic eye (Fig. 6).

On the frontal view film, if the eye was looking straight ahead while an $x$-ray was taken, the image of 

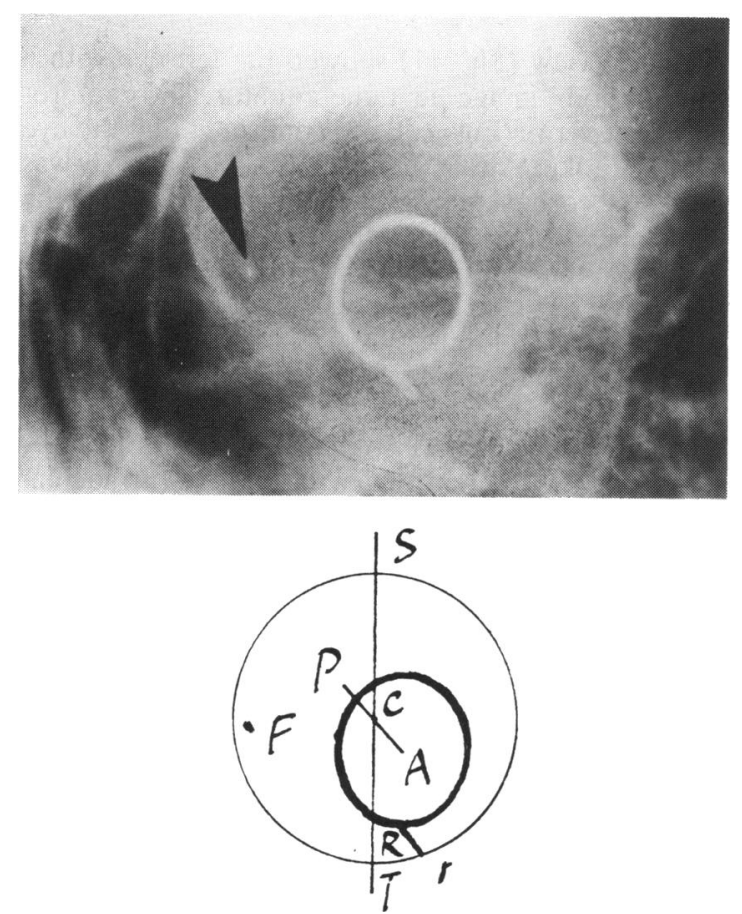

Fig. 12 The foreign body $\mathrm{F}$ is situated at the $90^{\prime}$ 'clock position.
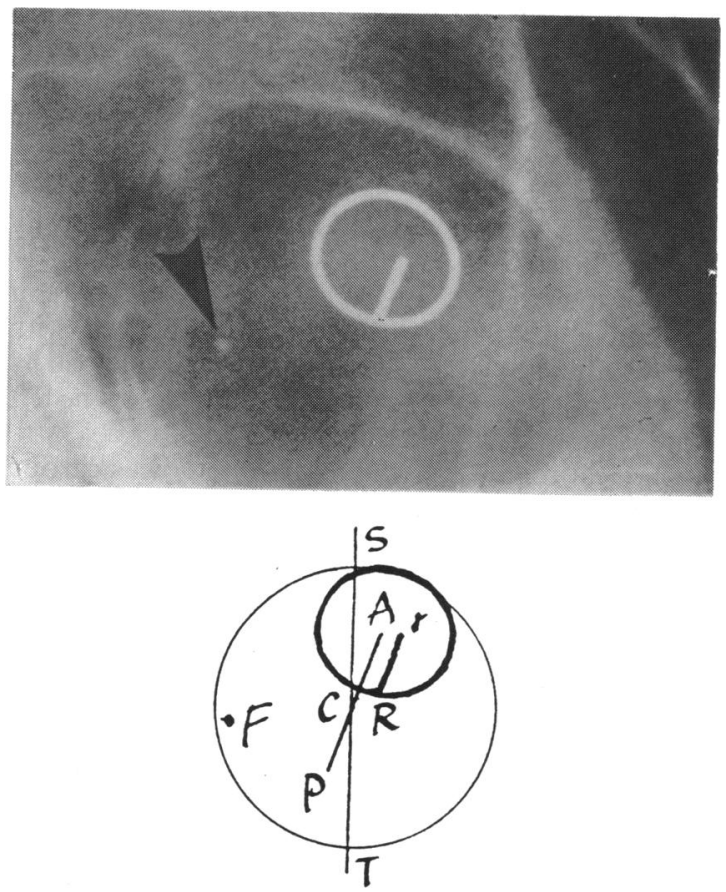

Fig. 13 The foreign body $\mathrm{F}$ is situated at the 90 'clock position.

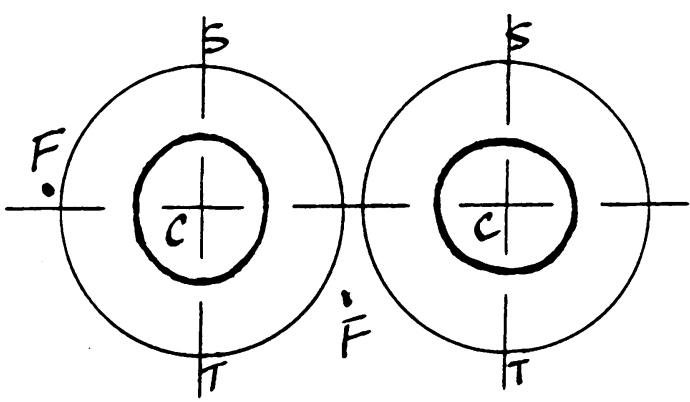

Fig. 14 If a limbal ring is used without a rod, the foreign body $\mathrm{F}$ cannot be located.

the rod would appear as a dot, and the corneal axis is also a dot. Hence all coronal tomographic planes (different diameter ST) appear as circles concentric with the ring. In practice, however, these films are rarely seen (Fig. 7).

The images of the rod usually appear with different directions and length, indicating that the eye was not looking straight ahead while the $x$-ray was being taken. A line is drawn parallel to the rod $r R$ through $A$, the centre of the ring, and the length AP is made equal to twice the length of the rod $\mathrm{rR}(\mathrm{AP}=2 \mathrm{rR})$. The mid point $\mathrm{C}$ of $\mathrm{AP}$ is the centre of the schematic eye (Fig. 8).

Next, the point $C^{\prime}$ on AP is marked so that the ratio of the lengths $A C^{\prime}: A P$ is identical to that in the lateral view shown in Fig. 6 . With $\mathrm{C}^{\prime}$ as the centre a circle is drawn with radius $\mathrm{SC}^{\prime}$ measured from the lateral view as in Fig. 6. The coronal tomographic plane where the foreign body (or bodies) $F(s)$ lies is produced (Fig. 9).

\section{Results}

If the foreign body $\mathrm{F}$ is situated inside the coronal tomographic plane of the schematic eye, it is intraocular foreign body; if outside the schematic eye, it is an extraocular foreign body. The foreign body can be located by the geometric drawings.

\section{Discussion}

As regards the image of the rod, we have information about the direction and the corresponding length of the anteroposterior axis of the schematic eye. Hence the coronal tomographic plane where the foreign body is situated can be constructed by the proportional geometric drawing method. Whatever the direction of the rod, the position of the foreign body in the coronal tomographic plane remains unchanged. If the limbal ring was used without the rod, only the centre of the ring could be used to construct the coronal planes; an intraocular foreign 
body would be misinterpreted as an extraocular foreign body, and vice versa (Fig. 10).

It should be noted that the size of the schematic eye is related to the size of image of the ring. Thus the influence of radiological magnification ${ }^{1247}$ on the localisation of an ocular foreign body can be eliminated (usually the magnification is $15-30 \%$ ).

\section{Case reports}

Two cases are reported to illustrate the localisation method.

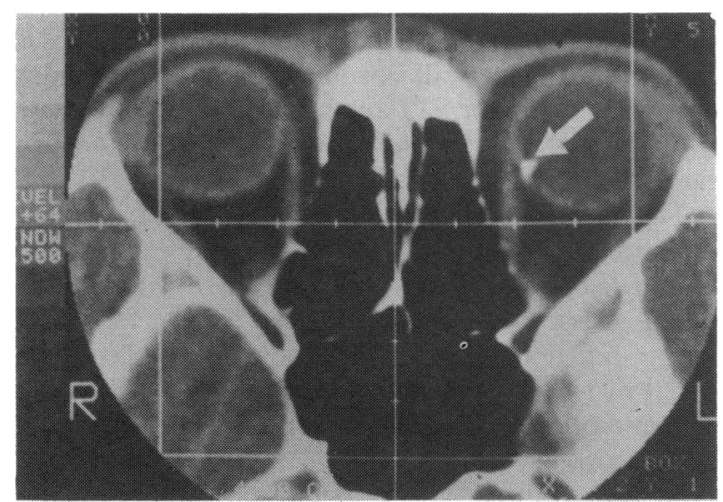

Fig. 15 CT scan.
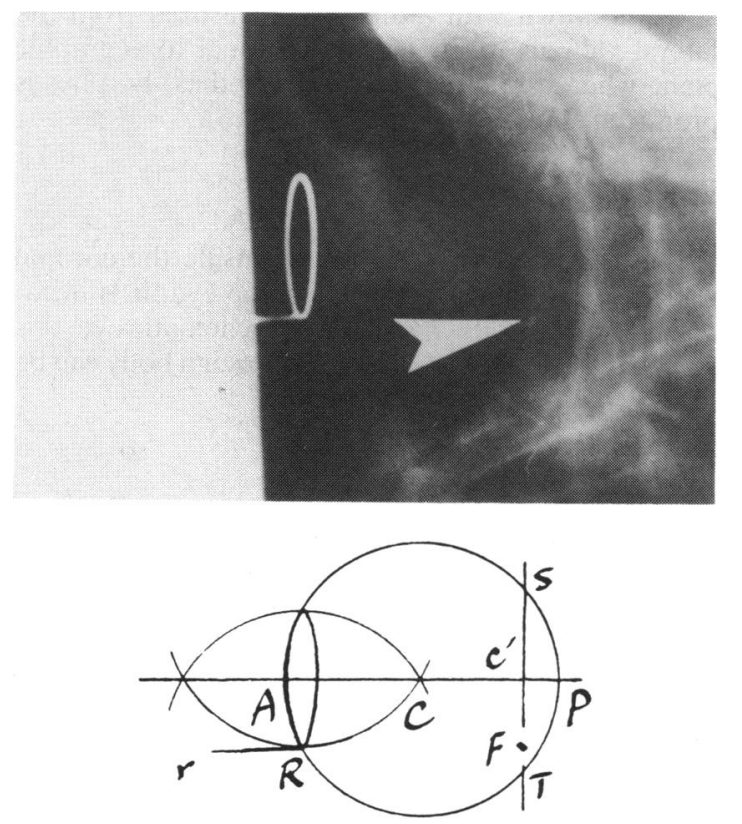

Fig. 16 The foreign body $\mathrm{F}$ is situated near the posterior pole $\mathrm{P}$.
CASE A

A lateral view (Fig. 11) showed the left eye with a foreign body image near the equator. Two anteroposterior views (Figs. 12,13) confirmed that the eye was not looking straight ahead. The foreign body was inside the coronal tomographic plane of the eye at the 9 o'clock position, near the wall. It is clear that, whatever the direction of the rod, the position of the foreign body in the coronal plane remains unchanged. If a limbal ring was used without a rod, Figs. 12 and 13 would appear as in Fig. 14. The foreign body $F$ could not be localised because the anteroposterior axis AP could not be found.

A radiological report can be made: Intraocular foreign body in left eye near the equator at the 9 o'clock position near the wall. A CT scan, ${ }^{37}$ horizontal section (Fig. 15) confirmed the presence of the foreign body. A blood clot in the vitreous at the 9 o'clock equator was also evident.

Surgical removal of the foreign body was carried out with a magnet through a sclerotomy at the equator at the 9 o'clock position.

\section{CASE B}

A lateral view (Fig. 16) showed the image of a foreign body in the left eye near the posterior pole. Two anteroposterior views (Figs. 17, 18) showed that the foreign body was inside the coronal plane of the schematic eye at the 6 o'clock position near the wall. If the limbal ring was used without a rod, Figs. 17 and 18 would appear as in Fig. 19. The foreign body F could not be localised.

A radiological report can be made: Intraocular foreign body in the left eye near the posterior pole at the 6 o'clock position near the wall. A CT scan, horizontal and coronal sections (Fig. 20), incorrectly showed that the foreign body was near the anterior pole. The error was due to the fact that the patient was looking upwards when the scan was taken, with the result that the lens shadow was in the same plane as the foreign body image in coronal section. The left fundus showed a blood clot in the vitreous.

Surgical removal of the foreign body was carried out by means of a posterior vitrectomy.

\section{Conclusion}

The advantages of the limbal ring-rod and the proportional geometric drawing method for localising ocular foreign bodies are, firstly, accuracy. This method allows the coronal plane to be obtained from a schematic eye, thus providing the information obtained from orbital tomography. From the image of the rod the direction and apparent length of the anteroposterior axis of the eyeball can be constructed in a way that is not affected either by movement of the eyeball or by $x$-ray magnification. 

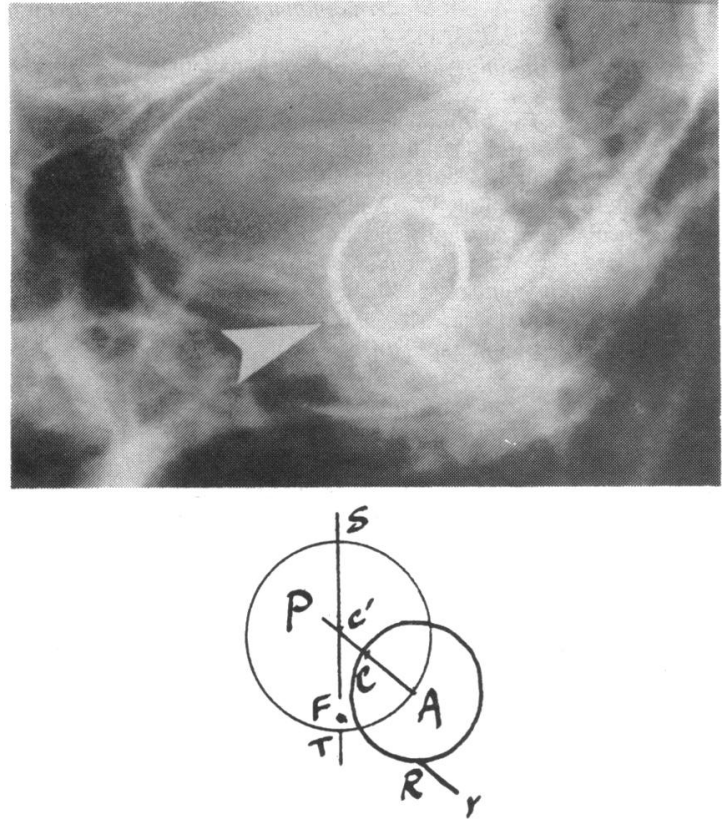

Fig. 17 The foreign body $\mathrm{F}$ is situated at the $6 o^{\prime}$ 'clock position.
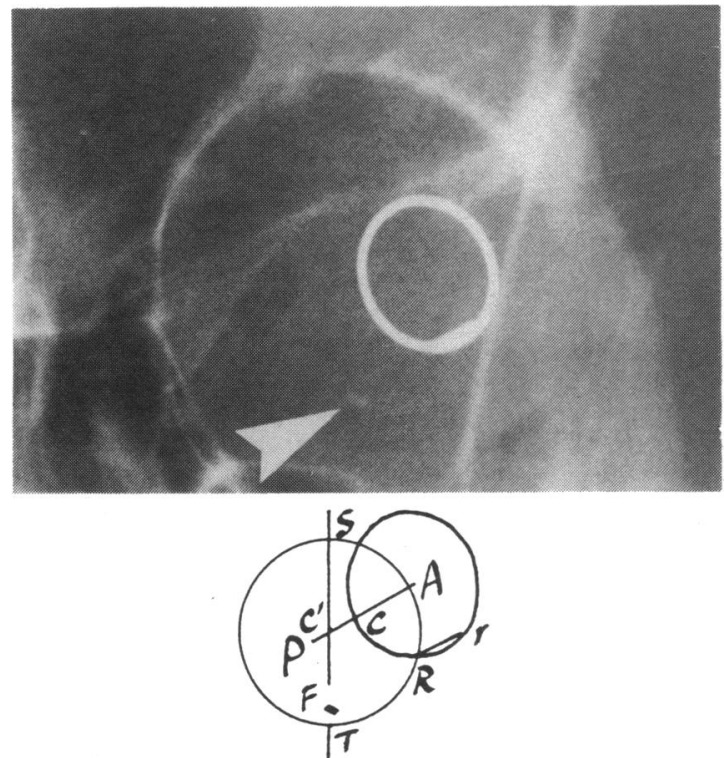

Fig. 18 The foreign body $\mathrm{F}$ is situated at the 6 o'clock position.

However, the accuracy could be reduced as follows: (1) Inaccurate transfer of images from the $x$ ray film on to paper. (2) Loosening of the stitches holding the limbal ring-rod on to the limbus. If the stitches become loose, the rod will move away from

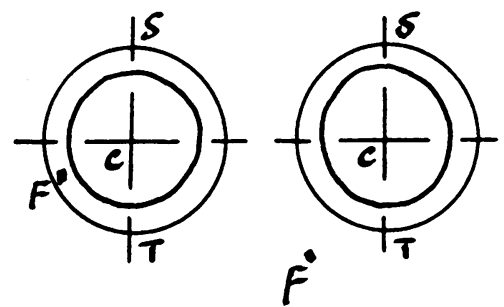

Fig. 19 If a limited ring is used without a rod, the foreign body F cannot be located.

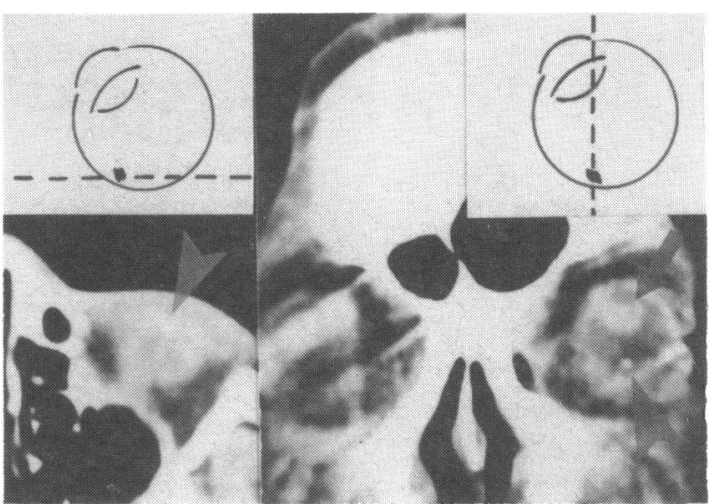

Fig. 20 CT scan. The patient was looking upwards when the scan was taken, with the result that the lens shadow is in the same plane as the foreign body image in coronal section. An intraocular foreign body in the posterior vitreous of left eye would be misinterpreted as in anterior vitreous.

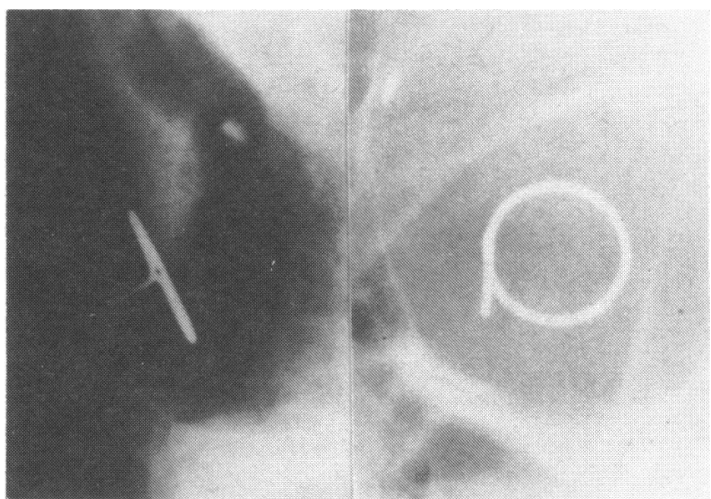

Fig. 21 Loosening of the stitches holding the limbal ringrod on to the limbus.

the 6 o'clock position; this will be shown on the $x$-ray films (Fig. 21). (3) The diameter of the patient's eye may be different from that of the schematic eye.

Secondly, the method is simple and inexpensive. This method allows foreign bodies to be localised and hence removed in poorly equipped hospitals. 
I thank the Hong Kong Government Ophthalmic Service for providing facilities for the development of this method, and I also thank Dr T K C Liu, consultant ophthalmologist, for his support of this work.

\section{References}

1 Duke-Elders, MacFaul PA. Mechanical injuries: the diagnosis and localization of intraocular foreign bodies. In: Duke-Elders ed. System of ophthalmology. London: Kimpton, 1972; 14: 565613.

2 Stallard HB. Eye surgery. 6th ed. Bristol: Wright, 1980: 775-83.

3 Cooling RJ. Ocular injuries: intraocular foreign bodies. In: Miller S, ed. Clinical ophthalmology. Bristol: Wright, 1987: 372-3.

4 Roper-Hall MJ. Injuries with retained foreign bodies: localiza- tion. In: Sorsby A, ed. Modern ophthalmology. 2nd ed. London: Butterworths, 1972: 3: 462-8.

5 Lloyd GAS. Radiology of the orbit. London: Saunders, 1975: 197210

6 Lloyd GAS. The orbit and eye: localisation of foreign bodies in the eye. In: Sutton D, ed. A textbook of radiology and imaging. 3rd ed. Edinburgh: Churchill Livingstone, 1980: 1006-11.

7 Goldberg MF, Paton D. Ocular emergencies: foreign bodies: intraocular and intraorbital. In: Peyman GA, Sanders DR. Goldberg MF, eds. Principles and practice of ophthalmology. Philadelphia: Saunders. 1980: 3: 2444-66.

8 Paton D, Goldberg MF. Management of ocular injuries. Philadelphia: Saunders, 1976: 99-162.

Accepted for publication 24 November 1988. 